\title{
Propuesta metodológica para articular la gestión documental con los requisitos de la Ley General de Archivos y la norma técnica internacional ISO 9001:2015
}

Methodological proposal to articulate the documental management of the Ley General de Archivos requirements and technical internacional standard ISO 9001:2015

Proposta metodológica para vincular a gestão documental com os requisitos da Lei Geral de Arquivos e a norma técnica internacional ISO 9001:2015

Maribel Puentes Poloche**

Fondo de Prestaciones Económicas, Cesantías y Pensiones - FONCEP

\footnotetext{
* Artículo de resultado de investigación. DOI: http://dx.doi.org/10.15332/s2145-1389.2017.0002.05

** Magíster en Calidad y Gestión Integral. Especialista en Archivística. Historiadora. Historiadora en el Fondo de Prestaciones Económicas, Cesantías y Pensiones - FONCEP. Correo electrónico: maribelpuentespoloche@hotmail.com
} 


\section{RESUMEN}

El objetivo de esta investigación fue elaborar una propuesta metodológica que permitiera articular la gestión documental cumpliendo con la Ley General de Archivos con la norma técnica de calidad ISO 9001:2015. Lo anterior, con el ánimo de que cualquier organización en Colombia implemente ambos sistemas sin que en la práctica surjan inconvenientes relacionados con el origen de la gestión documental como función archivística y el Sistema de Gestión de la Calidad como un enfoque por procesos. Como resultado de esta investigación, se hizo la elaboración de una propuesta metodológica para la articulación de la gestión documental con el Sistema de Gestión de la Calidad teniendo en cuenta los niveles estratégico, técnico y humano y, al interior de cada uno de ellos el ciclo PHVA. Además, un anexo para la alta dirección, quien toma las decisiones estratégicas y un segundo documento a coordinadores o personal encargado de lineamientos en materia documental y de gestión de calidad. Finalmente, la validación por juicio de expertos para que con sus conocimientos y experiencia se hiciera la versión final de la propuesta. La escogencia de la oficina del Convenio USTA-ICONTEC se hizo por el cumplimiento de las siguientes condiciones: implementación de ambos sistemas, fácil acceso a la documentación e información y la colaboración de funcionarios encargados de los lineamientos de calidad y de archivo. Sin embargo, la metodología propuesta es aplicable a cualquier otra entidad pública o privada que quiera implementar de manera articulada el Sistema de Gestión de la Calidad y la gestión documental, la cual debe cumplir con los requisitos de la Ley General de Archivos.

Palabras clave: gestión de calidad, gestión documental, Ley General de Archivos, NTC ISO 9001:2015, propuesta metodológica.

\section{ABSTRACT}

The objective of this research was to develop a methodology that would articulate the document management complying with the general law of files with the technical standard ISO 9001: 2015. This, with the intention that in Colombia any organization can implement both systems without inconveniences related to the source records management and archival function and Quality Management System as a process approach. As a result, the methodological proposal was made in three different scenarios: first, to the senior management, who take the strategic decisions; a second, coordinators or personnel who make guidelines to records and quality management. Finally, the proposal to the auxiliaries or employers, who have responsibility for carrying out the operational part and technical activities in the implementation of both systems. Meanwhile, USTA-ICONTEC agreement was chosen like the organization to make the diagnostic and interviews due to the implementation to the both system, easy access to documentation and information and collaboration to the employers who make Quality and Records guidelines. However, the proposed methodology can be applicable to another public or private entities wants or have to articulate the implementation between Quality and Records Management; which obey the requirements of the Ley General de Archivos.

Keywords: Quality management system, records management, NTC ISO 9001:2015, methodologic propose.

\section{RESUMO}

O objetivo desta pesquisa foi desenvolver uma metodologia que permitisse articular a Gestão Documental 
em conformidade com a lei geral de arquivos com a norma técnica de qualidade ISO 9001:2015 arquivos. Os dados anteriores têm o objetivo de que qualquer organização na Colômbia implemente ambos sistemas sem que na prática surjam problemas relacionados com a origem, a gestão documental como função de arquivamento e o Sistema de Gestão de Qualidade focados nos processos. Como resultado desta pesquisa elaborou-se uma proposta metodológica para a articulação da gestão documental com o sistema de gestão de qualidade levando em conta os níveis estratégicos, técnicos e humanos, e dentro de cada um deles o Ciclo PHVA. Também os anexos a diretores, que tomam decisões estratégicas e um segundo a coordenadores ou pessoal encarregado das normas em relação à Gestão Documental e de Qualidade. Finalmente, a aprovação por especialistas para que com seus conhecimentos e experiência se elaaborasse a versão final da proposta. A escolha da instituição do Convênio USTA-ICONTEC se fez pelo cumprimento das seguintes condições: implementação de ambos sistemas, fácil acesso à documentação e informação e a colaboração dos funcionários encarregados das normas de Qualidade e Arquivo. No entanto, a metodologia proposta é aplicável a qualquer entidade pública ou privada, que pretenda implantar de maneira articulada o sistema de Gestão de Qualidade e a Gestão Documental; o qual deve cumprir com os requisitos da Lei Geral de Arquivos.

Palavras-chave: gestão da qualidade, gestão documental, Lei Geral de Arquivos, NTC ISO 9001:2015, proposta metodológica.

\section{INTRODUCGIÓN}

El Sistema de Gestión de la Calidad NTC ISO 9001 (ICONTEC, 2015), se ha convertido no solo en la metodología utilizada para garantizar la eficacia, eficiencia y efectividad en las entidades privadas, sino también en la herramienta de gestión sistemática y transparente incorporada por el Estado colombiano obligatoriamente en las entidades públicas, principalmente en la Rama Ejecutiva (Ley Nº 872, 2003). Simultáneamente, la gestión documental ha tenido un espacio importante consolidado en la Ley N 594 de 2000 o Ley General de Archivos, en la cual se enuncian los principios de los archivos y de la función archivística orientada hacia la organización, conservación y preservación de la información institucional, no solo para la toma de decisiones, sino también para su recuperación, acceso y uso de la administración en el servicio al ciudadano como memoria institucional y fuente de la historia (Ley N $\left.{ }^{\circ} 594,2000\right)$.

Pese a que ambos sistemas están siendo implementados en Colombia, los principios en los cuales se fundamentan son diferentes: El Sistema de Gestión de la Calidad, hacia el enfoque al cliente, liderazgo, compromiso de personal, enfoque por procesos y mejora continua; mientras que la gestión documental, hacia la función archivística, organización jerárquica, ciclo vital del documento y el archivo total.

De la comparación entre estos principios cabe destacar el enfoque por procesos del Sistema de Gestión Documental y la función archivística de la gestión documental: el primero se aparta de la organización por dependencias de una estructura orgánica y se orienta a los procesos definidos como el conjunto de actividades mutuamente relacionadas o que interactúan, las cuales transforman elementos de entradas en resultados. Por su parte la función archivística está concebida por la Ley General de Archivos, como las actividades relacionadas con la totalidad del quehacer archivístico, que comprende desde la elaboración del documento hasta su eliminación o conservación permanente.

En Colombia, el cumplimiento de esta ley aplica para la administración pública en sus diferentes niveles y para las entidades privadas que cumplen funciones pú- 
blicas. La Superintendencia de Industria y Comercio (Resolución $\mathrm{N}^{\circ}$ 8934, 2014), estableció las directrices que en materia de gestión documental y organización de archivo debían cumplir los entes vigilados objeto de inspección, control y vigilancia por parte de esta Superintendencia. Por otra parte, en cualquier organización se hace necesario que la información documentada se organice y conserve de manera que sirva de evidencia de sus procesos y que sea de fácil acceso y pronta recuperación para la toma posterior de decisiones, debido a que los documentos en soporte físico, digital o electrónico, son el producto o evidencia del servicio entregado al cliente y se convierten en activos de la empresa para la continuidad del negocio.

A partir de la expedición de la Ley $\mathrm{N}^{\circ} 594$, el convenio entre el ICONTEC y el Archivo General de la Nación ha participado en la creación de la norma NTC 15489 y recientemente en la NTC 30301, cuya finalidad ha sido la de incorporar la gestión documental al Sistema de Gestión de la Calidad añadiendo los lineamientos del AGN con los encontrados en la NTC ISO 9001. Sin embargo, la articulación entre la norma de calidad y la ley de archivos tiene varias ambigüedades, ya que existen diferencias en los conceptos, alcance y finalidad de cada una de ellas por separado.

Una de las principales diferencias existentes fueron los conceptos de documento y registro aplicados en la norma ISO 9001:2008, con archivos y documentos de archivo de la Ley General de Archivos, ya que para la norma ISO, el registro eran documentos que presentaban resultados obtenidos o proporcionaban evidencia de actividades desempeñadas y los documentos, la información y su medio de soporte. No obstante, en el apartado 4.2, el control hacía referencia a los documentos del sistema y por separado el control de registro o de evidencias. Por su parte, para la gestión documental, tanto los registros como los documentos, hacen parte del concepto de archivo, ya que este fue establecido como el conjunto de documentos, sea cual fuere su fecha, forma y soporte material, acumulados en un proceso natural por una persona 0 entidad pública o privada, en el transcurso de su gestión, conservados respetando aquel orden para servir como testimonio e información a la persona o institución que los produce y a los ciudadanos, o como fuentes de la historia. Se entendió también a los documentos de archivo como el registro de información producida o recibida por una entidad pública o privada en razón de sus actividades o funciones.

Ahora bien, la norma ISO 9001:2015 unió los conceptos de documento y registro, en información documentada para mantener y conservar, pero dejó a la organización la libertad de definir qué conserva y qué mantiene, según sean sus necesidades. En este escenario, en la práctica no solo se debe definir qué se conserva y qué se mantiene, sino cómo y por cuánto tiempo, teniendo en cuenta el tipo de soporte. Parte de estas inquietudes han sido resueltas por el Estado a través de la Ley N 594 de 2000 o Ley General de Archivos, en la cual se dieron los principios de los archivos y de la función archivística orientada para disponer de una documentación organizada, de tal forma que la información institucional sea recuperable para uso de la administración en el servicio al ciudadano y como fuente de la historia.

Ante esta situación, surgió la necesidad de elaborar una propuesta que pueda responder a la pregunta sobre cómo articular la Ley General de Archivo al Sistema de Gestión de la Calidad. Para tal fin, se requirió tener acceso a una organización que tuviera implementado el Sistema de Gestión de la Calidad y la gestión documental con los lineamientos del AGN, para lo cual se acudió al convenio entre la Universidad Santo Tomás y el ICONTEC, ya que cumple con estos dos requisitos iniciales y por facilidad de acceso a los archivos y a los funcionarios que realizan ambas gestiones ${ }^{1}$.

1 Para acceder a la información documentada la autora firmó un acuerdo de confidencialidad con el convenio. 
En el estudio de las dos gestiones, Antonia Heredia, doctora en historia española, hace reflexiones sobre la gestión documental y gestión de calidad, sosteniendo que "son caras de la misma moneda y ambas han de ser el testimonio de la integración de los archivos en las instituciones, de su buen funcionamiento y como consecuencia del servicio esperado y demandado por cualquier usuario/cliente" (Heredia, 2011). Asimismo, la gestión documental debe entenderse como parte de la gestión administrativa que comienza en la creación de los documentos y va más allá de la exclusividad archivística.

En cuanto a América Latina, se han generado reflexiones sobre la implementación de los modelos de calidad que el Estado realiza en gestión pública con miras a incrementar el nivel de calidad para la prestación de sus servicios dirigidos no a un cliente, sino a la ciudadanía. Uno de los autores más estudiosos en esta materia es Jaime Torres Fragoso, quien en sus trabajos analiza la situación de la gerencia pública en los Estados latinoamericanos y su iniciativa por implementar modelos de calidad traídos de países más desarrollados como Estados Unidos y algunos de Europa (Torres, 2008). Propone además, la necesidad de que los gobiernos latinoamericanos replanteen los objetivos, las misiones, los valores, los procesos de atención y de servicio, la cultura organizacional imperante, etc. (pp. 53-54). De todos modos, y pese a los esfuerzos y compromisos adquiridos en los diferentes países, los resultados son poco favorables en la percepción de los usuarios, especialmente los que están relacionados con servicios públicos (X Conferencia Iberoamericana de Ministros de Administración Pública y Reforma del Estado, 2008).

Con respecto a Colombia, la mayoría de las publicaciones son cartillas o guías elaboradas por el Estado, las cuales se actualizan cuando la normatividad hace cambios en alguna de ellas. Una de estas es la Guía de Armonización Modelo Estándar de Control Inter- no MECI: 2005 y Sistema de Gestión de la Calidad NTCGP1000:2004, en donde se pretende que las entidades adquieran una mayor comprensión en la implementación de los sistemas.

De los estudios realizados al análisis entre la gestión documental y la calidad, sobresale el artículo de Luis Fernando Sierra, quien muestra los resultados de la gestión documental enfocada a procesos en la investigación realizada. Lo anterior hace parte de la adopción de normas de calidad de carácter internacional en la administración pública, a través de lineamientos como el Programa de Gestión Documental en el cual se detallan las operaciones para el desarrollo de los procesos documentales al interior de cada entidad, tales como producción, recepción, distribución, trámite, organización, consulta, conservación y disposición final de los documentos (Sierra, 2012, p. 244).

Bajo el enfoque de procesos, la gestión documental debe tener en cuenta tres elementos: el primero, es ir más allá de la identificación de la documentación para convertirse en la encargada de apoyar la implementación del Subsistema Interno de Gestión Documental y Archivos (SIGA); el segundo, es la administración electrónica aplicada a la tecnología de información indispensable para el alcance de la administración pública en cuanto a los planes Oficina cero papel y el Gobierno Abierto. Por último, la Gestión de la Calidad de la cual lo documental toma los procesos y específicamente los mapas de producción documental construidos a través de los mapas de procesos; los procedimientos, dentro de los cuales se identifican las actividades, y los registros, en donde se presentan los resultados, que para el caso de la gestión documental, son los manuales y guías (p. 249).

Así las cosas, las diferencias y ambigüedades de conceptos, alcance y método de implementación, se presentan al interior de la entidad cuando se realizan las actividades operativas para mantener y conservar la in- 
formación documentada cumpliendo los requisitos de ley al tiempo que se implementa el Sistema de Gestión de la Calidad. Por lo anterior, el objetivo de esta investigación es elaborar una propuesta de un modelo de gestión documental que dé respuesta en los requisitos de la Ley General de Archivos y a los requisitos de la norma ISO 9001 en el convenio USTA-ICONTEC.

Una vez se hizo elección de la organización, se pasó a desarrollar cada uno de los objetivos expuestos en el anteproyecto, los cuales fueron los siguientes:

- Análisis de la norma y la ley. Comparación entre las normas ISO 9001:2015, NTC 15489-1 y NTC 30301 con la Ley General de Archivos buscando los aspectos convergentes, divergentes y complementarios.

- Diagnóstico de la entidad. Este se dividió en dos partes: una, recopilación documental, y dos, entrevistas con los funcionarios tanto de la Universidad Santo Tomás como del ICONTEG encargados del Sistema de Gestión de la Calidad, de los lineamientos en materia de gestión documental.

- Elaboración de la propuesta metodológica a partir de los resultados de los dos objetivos anteriores. Se tuvieron en cuenta los niveles estratégico, técnico y humano y al interior de cada uno de ellos, las actividades del ciclo PHVA.

En esta propuesta se quiso realizar tanto el detalle metodológico para el personal operativo que realiza las actividades, como los objetivos y acciones para los coordinadores y el esquema de actividades y responsabilidad direccionado a la alta dirección, para tener una perspectiva más amplia y que abarcara a toda la organización. Adicionalmente, se espera que los resultados obtenidos y plasmados en la propuesta, se apliquen en cualquier entidad pública o privada que incorpore al Sistema de Gestión de la Calidad no solo otros sistemas de calidad, sino también la aplicación de los lineamientos de gestión documental que garantizan el manejo adecuado de la información, con las generalidades que pueden ser aplicadas a otras organizaciones públicas o privadas.

\section{METODOLOGÍA}

La investigación estuvo orientada hacia el análisis de contenido siguiendo las siguientes fases:

- Análisis de contenido. Utilización de fuentes primarias como entrevistas semiestructuradas de preguntas abiertas dirigidas a los responsables de la gestión documental y de la calidad y a los líderes de proceso. Para ello, se estableció un guion de entrevistas y una vez realizadas, se transcribieron cada una de ellas para analizarlas y codificarlas en el aplicativo ATLAS.ti.

- Diseño exploratorio. Estado actual de la gestión documental. Se hizo la revisión documental a una entidad dentro del convenio USTA-ICONTEC, teniendo en cuenta las variables descritas anteriormente, es decir, la implementación de la gestión documental conforme a la Ley General de Archivos y el Sistema de Gestión de la Calidad. Adicionalmente, se realizaron entrevistas semiestructuradas, en donde las preguntas fueron construidas a partir del establecimiento de las categorías: Gestión documental dentro de la cual se incluyeron las subcategorías de Función archivística y Programa de gestión documental; y Sistema de gestión de la calidad, donde las subcategorías fueron Políticas de calidad y Procesos.

- Triangulación técnica. La elaboración de la propuesta se hizo a partir de las conclusiones de las fases anteriores que dieron respuesta a los objetivos 1,2 y 3 de esta investigación. 
- Validación de la propuesta. Se hizo validación cognitiva por expertos en el tema de esta investigación, para que una vez ajustada, se lograra generar la propuesta final.

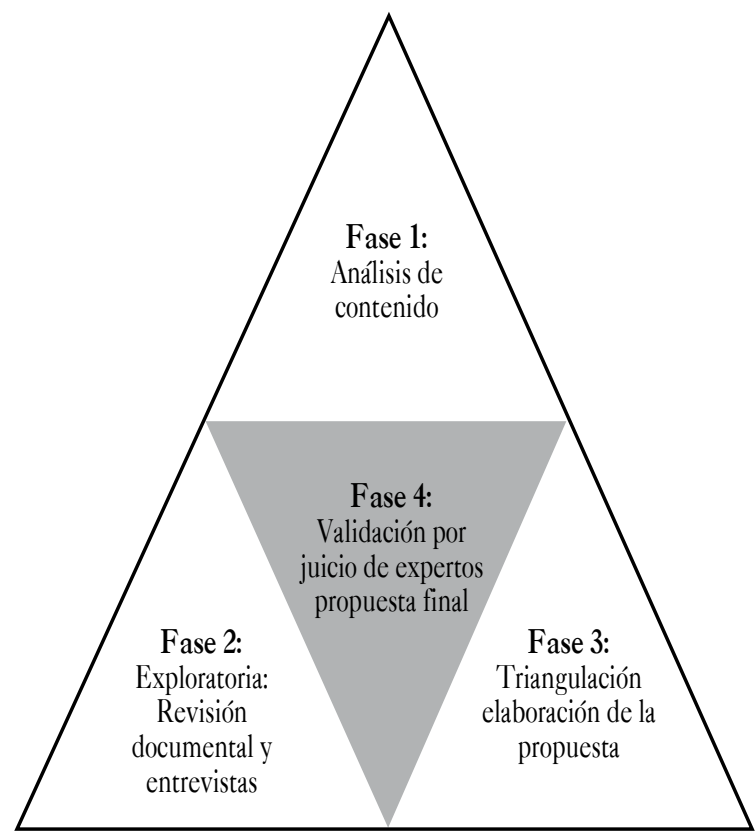

Figura 1. Fases de la investigación.

Fuente: la autora.

La metodología utilizada en esta investigación se inició con la clasificación de los aspectos a tener en cuenta para llegar a la elaboración de la propuesta metodológica. En primer lugar, con la identificación de las leyes y normas de la gestión documental en Colombia y en segundo término, con el Sistema de Gestión de la Calidad, en este caso, la Ley General de Archivos y la norma ISO 9001:2015 respectivamente, como ejes centrales.

De igual manera, se revisaron las normas técnicas colombianas $15489-1$ y 30301 ya que fueron elaboradas por el convenio existente entre el ICONTEC y el Archivo General de la Nación. En términos generales, se hizo un análisis de contenido para obtener como re- sultado una matriz que evidenciara los elementos de convergencia, otra con los de divergencia y una tercera con los de complementariedad entre la ley y la norma.

Para la elaboración del diagnóstico se tuvieron en cuenta dos aspectos:

Recopilación de la información: análisis de fuentes primarias de la entidad, es decir, acceso a los documentos de calidad (manual y procedimientos) e instrumentos archivísticos (tablas de retención documental) elaborados para el convenio como directrices a seguir y aplicar en materia de gestión documental y de calidad. Así mismo, se revisó el archivo producido por la oficina que llevaba los procesos y funciones del convenio.

Entrevistas semiestructuradas: las entrevistas fueron realizadas a los empleados y funcionarios encargados de dar los lineamientos para la gestión de calidad, la gestión documental y quienes operativamente realizaban la aplicación. Con respecto a la implementación del Sistema de Gestión de la Calidad, se entrevistó tanto a la coordinadora de calidad de la Universidad Santo Tomás como a la coordinadora del ICONTEC; en cuanto a la gestión documental, al coordinador de archivo de la Universidad Santo Tomás, quien elabora las tablas de retención documental y demás directrices del convenio, y en la parte operativa de la oficina del convenio, a la funcionaria encargada de dicha implementación, la cual se evidencia en los registros o documentos de archivo que están almacenados y conservados para la oficina que lleva el convenio.

Se transcribieron las entrevistas y se utilizó el software ATLAS.ti para la sistematización y análisis cualitativo de la información suministrada por los entrevistados, la cual fue codificada de acuerdo a las categorías y subcategorías mencionadas previamente. Las respuestas de los entrevistados y los resultados del diagnóstico arrojan información importante para la elaboración de la propuesta. 
De este resultado se evidencia que la propuesta tiene que ser planteada en tres escenarios para que su descripción tenga un mayor alcance, así:

1. Alta dirección o quienes toman las decisiones estratégicas de la entidad, instancias fundamentales para que tenga éxito la implementación articulada de ambas gestiones.

2. Coordinadores o responsables de dar los lineamientos en gestión documental y de calidad. Los instrumentos archivísticos y los procedimientos de calidad deben estar alineados para que sean complementarios y orienten en una misma dirección a todo el personal de la entidad.

3. Auxiliares o responsables de ejecutar las actividades. Al interior de las organizaciones se encuentra personal que se encarga de la administración y conservación de los documentos de archivo. Estas personas al igual que toda la organización, deben recibir capacitación en cuanto a conceptos e instrucciones, ya que de un modo u otro tienen responsabilidades con los documentos que evidencian su gestión.

Por otra parte, para la elaboración de la propuesta metodológica se tomó el ciclo PHVA con la implementación de tres niveles, así:

- Estratégico. Se determinaron las acciones estratégicas a realizar en cada una de las fases del ciclo PHVA, es decir, toma de decisiones para la implementación de ambas gestiones, asignación de recursos, planteamientos a desarrollar y en fin, las acciones a realizar en el ciclo (planear, hacer, verificar y actuar).

- Técnico. La parte técnica fue vital para el desarrollo de la implementación, debido a que en ella se describieron las herramientas y procedimientos a diseñar, y el seguimiento y monitoreo que se debe realizar para garantizar la implementación y articulación satisfactoria.

- Humano. Recurso más importante al interior de las entidades para realizar esta implementación, que incide en el éxito o fracaso de la misma.

Teniendo en cuenta lo anterior, se partió de las cuatro reglas establecidas en la norma internacional de descripción archivística ISAD $(G)$, en la cual, el fondo de una entidad se mira como un todo en donde se describe cada una de sus partes por separado (Consejo Internacional de Archivos, 2000); estas reglas son:

a) Descripción de lo general a lo particular.

b) Información pertinente para el nivel de descripción. Proporcionar solo aquella información adecuada al nivel que se está describiendo.

c) Vinculación de las descripciones. Vincular cada descripción con la unidad de descripción inmediatamente superior.

d) No repetición de la información. Evitar información redundante.

La validación de la metodología se realiza por juicio de expertos para posteriormente ajustar y actualizar con las observaciones de los mismos. Los criterios utilizados fueron los siguientes: 1) claridad, el punto se comprende fácilmente, es decir, su sintáctica y semántica son adecuadas; 2) coherencia, el ítem tiene relación lógica con la dimensión o indicador que está midiendo; 3) relevancia, el tema es esencial o importante, es decir debe ser incluido; $y, 4)$ suficiencia, los ítems que pertenecen a una misma dimensión bastan para obtener la medición de esta. 


\section{RESULTADOS Y DISCUSIÓN}

Para la Fase 1 (ver Figura 1), el análisis comparativo dio como resultado matrices que indicaban convergencias, entendidas como las similitudes entre la ley y la norma frente a los estándares manejados y que son importantes para mostrar su finalidad, principios y lineamiento; divergencias entre la Ley General de Archivos y la norma de calidad, las cuales han ocasionado implicaciones en la forma como la organización concibe los deberes y obligaciones con la ley os. los beneficios y responsabilidades que trae la norma. Finalmente, complementariedad, en cuanto a lo que tiene la ley y que complementa la norma o viceversa.

De este análisis se concluye que la gestión documental y el Sistema de Gestión de la Calidad en Colombia, han estado relacionados permanentemente aunque con enfoques diferentes. En el caso de la gestión documental, su reglamentación se hizo mediante documentos de orden legal a entidades públicas principalmente, en donde se dice el qué, pero no cómo hacen las empresas para dar cumplimiento a estos requisitos de ley. El Estado a través del Archivo General de la Nación vigila y controla, más que proponer metodologías que garanticen la implementación de la Ley General de Archivos, la cual después de más de 15 años de haberse promulgado, no ha sido reglamentada en su totalidad.

En contraste con lo anterior, el Sistema de Gestión de la Calidad se implementó a través de una norma internacional de carácter voluntario y enfocado a la satisfacción del cliente, las partes interesadas, las buenas prácticas y a la mejora continua en las entidades, principalmente las privadas. Adicionalmente, el Estado adopta el Sistema de Gestión de la Calidad para las entidades públicas. Ya en el desempeño institucional, la gestión documental y la calidad se encuentran con diferentes puntos de vistas, dentro de los cuales se destacan: la calidad entendida como una gestión por procesos, que articula las áreas y está encaminada hacia el cliente, mientras que la gestión documental entendida como una función archivística, es una estructura orgánica dividida por dependencias. Sin embargo, el AGN y el ICONTEC han unido esfuerzos para que, mediante normas técnicas colombianas, la gestión documental haga parte del Sistema de Gestión de la Calidad; en un primer momento, en la norma ISO 15489-1 con un orientación más hacia la función archivística y en la norma ISO 30301 basada en una estructura de alto nivel, en donde se adelanta a la norma ISO 9001:2015 al incluir el contexto como uno de los numerales que componen la norma.

Seguidamente al análisis de la ley vs. la norma, es fundamental la elaboración de un diagnóstico, ya que para la implementación de la gestión documental y del sistema de calidad se debe conocer previamente el estado actual de la organización, lo cual permite identificar las características particulares de cada institución, ya que estas inciden en los elementos que se deben subrayar a la hora de tomar decisiones para la implementación de la gestión documental. En este caso, al ser un convenio, los lineamientos de las dos entidades confluyen en la oficina productora ocasionando ambigüedades en el momento de realizar el desarrollo de actividades para la implementación de la gestión documental.

Para la Fase 2, las entrevistas arrojaron que la Universidad Santo Tomás y el ICONTEC cuentan con personal calificado para la elaboración e implementación del Sistema de Gestión de la Calidad y están en el proceso de actualización de la norma ISO 9001:2008 a la ISO 9001:2015, reconociendo las beneficios que traerá esta última versión, especialmente el cambio de registros y documentos a información documentada y la inclusión del contexto como apartado de la norma. Por su parte, para la Ley General de Archivo, la USTA tiene solamente un profesional en bibliotecología y archivística 
especializado en calidad, quien no solo cumple con la ley sino también con la elaboración de herramientas archivísticas como son las tablas de retención documental. Además, articula ambas gestiones cuando en los tipos documentales de cada una de las series incluye tanto la ubicación física o digital, como los formatos encontrados en los procedimientos de todos los procesos.

En cuanto al ICONTEC en materia documental, sigue los lineamientos de la Universidad en esta materia, pero no cuenta con profesionales con formación 0 auxiliares capacitados, sino que la organización de los archivos hace parte de una de las funciones asistenciales y falta tiempo suficiente para la finalización de todas las actividades hacia la disposición final de los documentos. Esta situación afecta la realización de las actividades complementarias a la organización, como la elaboración de inventarios y las transferencias documentales al Archivo Central.

Para la Fase 3, se determinó la elaboración de la propuesta metodológica para la implementación de ambas gestiones como instrumento de cohesión al interior de la organización, con la optimización del sistema de gestión integral de archivo, identificación de sus fortalezas, detección de errores y corrección de las causas que los han producido; lo anterior conlleva a facilitar el acceso a la información. Los siguientes fueron los resultados al incorporar al ciclo PHVA, los niveles estratégico, humano y técnico:

- Estratégico. Actividades a realizar en cada una de las fases del ciclo PHVA, que partían de la toma de decisiones para la implementación de ambas gestiones relacionadas con asignación de recursos, planteamientos a desarrollar y en fin, las acciones a realizar en el planear, hacer, verificar y actuar.

- Técnico. La parte técnica se hizo esencial para el desarrollo de la implementación, debido a que en ella se describieron los instrumentos y procedimientos a diseñar, el seguimiento y monitoreo realizado para garantizar la implementación y articulación satisfactoria y la orientación hacia la mejora continua siguiendo una misma dirección para ambas gestiones.

- Humano. Este es el recurso o soporte más importante al interior de las entidades para realizar esta implementación e incide en el éxito o fracaso de esta. En cada una de las fases se requiere de liderazgo, capacidad, idoneidad y cultura organizacional para llegar a la gestión del cambio.

Como parte de los resultados de la triangulación técnica del análisis de contenido de las normas técnicas y reglamentarias con los obtenidos en la revisión documental primaria y secundaria, es la información anexa a la propuesta, así: a) presentación ejecutiva a la alta dirección o a quienes toman las decisiones estratégicas de la entidad, fundamental para la obtención de los recursos y el compromiso con la aprobación y aplicación de la propuesta $y, b)$ ciclo PHVA para coordinadores o responsables de dar los lineamientos en gestión documental y de calidad, encargados de la planeación y ejecución para que la implementación tenga éxito en el corto, mediano y largo plazo.

De esta manera, el detallado de las actividades a realizar incluye los trabajadores de toda la entidad, tanto quienes tienen funciones de archivo como aquellas personas que por su quehacer administrativo, participan en la conformación de los expedientes y, por ende, de los archivos de la entidad. No obstante, debido a la importancia de organizar y custodiar la documentación desde su fase inicial, se requiere que se tengan reseñadas las acciones junto con las actividades y resultados en cada una de las fases del ciclo PHVA, cuyo alcance es la gestión del cambio, en donde la implementación de ambas gestiones haga parte de la eficacia, eficiencia y efectividad de la gestión organizacional. 
En general, con la aplicación se quiere mejorar el sistema de gestión integral del archivo, identificar sus fortalezas, detección de errores y corrección de las causas que los han producido, a partir de simplificar labores en el manejo y control de la documentación en cualquier entidad, facilitando el control de la documentación, el acceso de manera inmediata y evitando los riesgos de pérdida. Adicionalmente, la documentación como soporte del Sistema de Gestión de la Calidad, garantiza la operación de la organización, el desarrollo de los procesos, la toma de decisiones y la recuperación de la información o registros que evidencien la gestión.

Dentro de los anexos, el esquema de responsabilidades y actividades se dirige a la alta dirección, debido a que esta instancia es quien decide si se implementan 0 no ambos sistemas de acuerdo a las necesidades o el alcance que quiera tener la entidad. Por lo anterior, para involucrarla y que sepa cómo se va a realizar la implementación, es necesario presentar la metodología de manera concisa, pero que incluya las actividades a desarrollar, responsables de los lineamientos y las partes involucradas, una vez se decida tomar tres decisiones simultáneas y fundamentales para la implementación, a saber: 1) alinear la gestión documental con el Sistema de Gestión de la Calidad, 2) impartir responsabilidades y compromisos en todos los niveles y, 3) reconocer la importancia de la gestión documental como soporte a la gestión de calidad.

En cuanto a los coordinadores y personal encargado de dar los lineamientos, la metodología está orientada hacia la totalidad de las actividades en los niveles estratégico, técnico y humano en cada una de las etapas del ciclo PHVA. Con lo anterior, los coordinadores pueden tener un conocimiento completo de todas las actividades a realizar en el corto, mediano y largo plazo, lo cual es de vital importancia para ellos, ya que son los que tienen a cargo la ejecución de la implementación y el garantizar que se realice conforme a lo determinado por la alta dirección.
En la gestión de calidad como sistema integral, se debe lograr la consolidación en las diferentes esferas de la organización, incorporando procesos transversales y de apoyo, los cuales son indispensables para el clima organizacional y el liderazgo a nivel local, regional e incluso internacional. En cuanto a la gestión documental, como lo entienden los profesionales del convenio y de la Universidad Santo Tomás, no van en contravía las dos gestiones, ambas hacen parte de la gestión organizacional, son complementarias y esta separación está más dada por las técnicas de aplicación diferentes entre la una y la otra, pero que al ser el Sistema de Gestión de la Calidad una metodología que abarca el manejo de toda la organización, es preciso que la gestión documental se incorpore como herramienta que garantice la administración, conservación y preservación de la información documentada decisiva en la toma de decisiones, satisfacción al cliente y continuidad del negocio.

La validación del contenido de esta propuesta metodológica (Fase 4), se hizo como parte de su proceso de estimación y el juicio de expertos amplió la metodología en cuanto a su desarrollo.

La propuesta evidenció que en las entidades se cumple con los lineamientos de ambas gestiones reglamentadas por la ley y por la norma encontrada para tal fin; sin embargo, operativamente, al llevarse a cabo por separado hace que los instrumentos generados para gestión documental y para calidad no sean consistentes y contengan información diferente: un ejemplo son las tablas de retención documental con los registros de los procedimientos en los procesos de calidad.

El objetivo trazado en las entidades no debe ser solo el cumplimiento de la ley o de la norma, sino que este debe ir orientado a simplificar las actividades en el manejo y control de la documentación en las organizaciones, en las cuales las entradas y salidas de su gestión son documentos de archivo o información documenta- 
da independiente de su soporte. Lo anterior, se traduce en garantizar la adecuada realización de las actividades en los procesos de organización y conservación de los archivos, la reducción de tiempos, optimización de recursos y la satisfacción de los usuarios.

Con respecto a las entidades públicas o con funciones públicas, no solo estas lo aplicarán como plan de mejora o buenas prácticas en su Sistema de Gestión de la Calidad, sino que además, cumplirán con las directrices de la Ley General de Archivo y de la NTCGP 1000:2009. Lo anterior, además de incidir en su gestión administrativa, también se verá manifestado directamente en la prestación de sus servicios y la satisfacción de los usuarios.

Ahora bien, pese a que el diagnóstico se realizó en el convenio USTA-ICONTEC, no se puede considerar que sea un estudio de caso ni que la propuesta de esta metodología sea aplicable solo en esta entidad, debido a que la gestión documental y el sistema de calidad son regulados por lineamientos legales y estándares técnicos internacionales, respectivamente. Lo anterior, denota la implementación conforme a las disposiciones establecidas en la Ley General de Archivos y a la norma técnica de calidad vigente sin alterar sus principios, alcances o finalidades.

En la práctica, para la articulación de ambas gestiones, se debe iniciar con la producción de los lineamientos en calidad, asociados a los instrumentos de la gestión documental, debido a que la información contenida en los instrumentos archivísticos debe hacer parte de los procedimientos y demás documentos para la gestión de la calidad. Por otra parte, el enfoque por procesos circunscribe las funciones que se manejan por parte de funcionarios que pueden pertenecer a una o varias dependencias y que condensan los asuntos macro de la misión y la visión institucionales.
Finalmente, para la continuidad de este proyecto se deben aprovechar los convenios del ICONTEC con otras instituciones académicas, como la Universidad de la Salle y entes reguladores como el Archivo General de la Nación, a través de acuerdos de cooperación para que se realice el paso siguiente a esta propuesta, que es una prueba piloto en una 0 varias instituciones para ajustar y proponer una guía a seguir en cualquier entidad. Esta prueba debe contemplar:

- Contexto de la organización.

- Situación interna de la implementación de la gestión documental y el Sistema de Gestión de la Calidad.

- Factores de convergencia, divergencia y complementariedad.

- Ajustes a la propuesta inicial.

- Plan de implementación con objetivos de corto, mediano y largo plazo.

- Resultados.

\section{CONCLUSIONES}

El resultado de esta investigación es una propuesta a las organizaciones para que la puedan aplicar en su gestión administrativa, tanto para la gestión documental como para el Sistema de Gestión de la Calidad integrándolos en un solo sistema de gestión, ya que esto es indispensable en las entidades de cualquier sector al ser la certificación importante para la competitividad y el aprovechamiento de los tratados internacionales.

Por tal motivo, la conclusión a presentar es la recopilación de las conclusiones parciales de los capítulos expuestos en el desarrollo de la investigación, y que en 
conjunto conllevaron a la elaboración de la propuesta metodológica para el alto nivel, para los coordinadores y para el personal de la entidad.

En el análisis de la comparación entre la Ley General de Archivos y la norma ISO 9001:2015, La gestión documental y el Sistema de Gestión de la Calidad en Colombia han estado relacionados permanentemente aunque con enfoques diferentes; en el caso de la gestión documental su reglamentación se hizo mediante documentos de orden legal a entidades públicas principalmente, en donde se dice el qué, pero no cómo hacen las empresas para dar cumplimiento a estos requisitos de ley. El Estado a través del Archivo General de la Nación vigila, y controla más que proponer metodologías que garanticen la implementación de la Ley General de Archivos, la cual después de más de 15 años de haberse promulgado, no ha sido reglamentada en su totalidad, por lo que los lineamientos en gestión documental están en permanente cambio, actualización y otros nuevos generalmente están relacionados con documentos electrónicos y digitales.

En contraste a lo anterior, el Sistema de Gestión de la Calidad se implementó a través de una norma internacional de carácter voluntario y enfocado a la satisfacción del cliente, las partes interesadas, las buenas prácticas y a la mejora continua en las entidades, principalmente las privadas. Adicionalmente, el Estado adopta el Sistema de Gestión de la Calidad para las entidades públicas y en el desempeño institucional, la gestión documental y la calidad se encuentran con diferentes puntos de vistas dentro de los cuales se destacan: la calidad entendida como una gestión por procesos, que articula las áreas y encaminada hacia el cliente, mientras que la gestión documental entendida como una función archivística, es una estructura orgánica dividida por dependencias. Sin embargo, el AGN y el ICONTEC han unido esfuerzos para que, mediante normas técnicas colombianas, la gestión documental haga parte del Sistema de Gestión de la Calidad; en un primer momento, en la norma ISO 15489-1 con un orientación más hacia la función archivística y en la norma ISO 30301 basada en una estructura de alto nivel, en donde se adelanta a la norma ISO 9001:2015 al incluir el contexto como uno de los numerales que componen la norma.

Por otra parte, Los recursos y componentes comunes en ambas gestiones se continúan analizando individualmente, por lo que en la propuesta se establece cuáles son y cómo se articulan en las instituciones para que finalmente, se orienten no solo a la complementación de los dos sistemas, sino que los dos hacen parte de la gestión de cualquier organización. Del mismo modo, el enfoque por procesos circunscribe las funciones que se manejan por parte de funcionarios que pueden pertenecer a una 0 varias dependencias y que condensan los asuntos macro de la misión y visión, lo cual tiene como entradas y salidas información documentada a mantener y conservar de acuerdo a las necesidades de la organización.

Por lo anterior, es indispensable para la articulación de ambas gestiones comenzar con la producción de los lineamientos en calidad y gestión documental, debido a que la información contenida en los instrumentos archivísticos deben ser el reflejo de los realizados para calidad; por ejemplo, las series y tipos documentales de las TRD deben ser igual a los registros que hacen parte de los procedimientos, instructivos y formatos. Aún más, una de las entradas para la elaboración de las TRD de las oficinas productoras, son los procedimientos identificados para cada uno de los procesos.

Los anexos de la propuesta no estaban considerados en el proyecto inicial, pero una vez realizadas las dos primeras fases, se evidenció la necesidad de elaborar un primer documento que facilitara la aprobación de la misma por parte de la alta dirección, y un segundo documento dirigido a los coordinadores para que tuvieran todas las actividades de los niveles en el ciclo PHVA 
con la finalidad de establecer los objetivos a corto, mediano y largo plazo.

Académicamente, se quiere ayudar a resolver las problemáticas de nuestro contexto con la utilización de un método científico con recopilación y análisis de la información confiables para su posterior implementación a nivel nacional. Además, trascender a nivel internacional, ya que en la actualidad estas dos gestiones son manejadas en otros países con finalidades equivalentes.

Para la implementación de esta propuesta debe tenerse en cuenta lo siguiente:

- El estado actual en que se encuentra la organización identificando sus factores internos y externos. En el diagnóstico, la revisión de los archivos da los resultados no solo de la organización de los archivos como tal, sino cómo se tiene el acceso y recuperación de la información documentada, así como su preservación a corto, mediano y largo plazo.

- Incluir los decretos reglamentarios de la Ley General de Archivos y actualizar la propuesta en caso de que se actualice la ley o se incluyan nuevos. De igual manera, cuando se actualice la NTC ISO: 9001:2015.

- Es importante que las actualizaciones realizadas en los procesos y procedimientos sean reportadas al personal de archivo para que también se realicen las modificaciones a los instrumentos archivísticos.

- La organización y administración de documentos debe realizarse desde la creación de los mismos, lo cual hace que se involucre a todo el personal de la organización según sus actividades. Cuando esta función la desarrolla una sola persona, hay represamientos y cumplimiento parciales de las labores.

- La resistencia a seguir los lineamientos de gestión documental se presenta en todos los niveles, por lo tanto el apalancamientos por parte de la alta dirección es decisivo para la gestión del cambio.

- Se deben tener recursos físicos, humanos y técnicos para la implementación de la gestión documental, debido a que no solo se está hablando de los documentos, sino también de los depósitos de archivos.

- Las entidades deben estar direccionadas hacia la gestión de archivos digitales y electrónicos con el uso de los avances tecnológicos para su implementación.

- La gestión del cambio y la cultura del personal interno e incluso de las partes interesadas debe hacer parte de la implementación.

- La gestión documental y la gestión de calidad están circunscritas en la gestión organizacional que tienen las entidades y que articuladas con otras, facilitan la administración, el cumplimiento de la misión y visión y el funcionamiento de las organizaciones. En el caso del Sistema de Gestión de la Calidad, incluye a toda la entidad, incluyéndose allí la gestión documental, la cual administra la organización de los archivos o evidencia de la gestión.

\section{REFERENCIAS}

Consejo Internacional de Archivos. (2000). Comité Ad Hoc de Normas de Descripción. ISAD. (G): Norma Internacional General de Descripción Archivística. Madrid, España: Subdirección de los Archivos Estatales.

Heredia, A. (16 de abril de 2011). Gestión documental y calidad. Mundo Archiviśtico [en línea]. < http:// www.mundoarchivistico.com/?menu=articulos\&id=212> 
Propuesta metodológica para articular la gestión documental con los requisitos de la Ley General de Archivos y la norma técnica internacional ISO 9001:2015

ICONTEC. (2015). NTC ISO 9001:2015. Bogotá D.

C., Colombia: ICONTEC.

Ley $\mathrm{N}^{\circ}$ 872. Por la cual se crea el sistema de gestión de la calidad en la Rama Ejecutiva del Poder Público y en otras entidades prestadoras de servicios. Diario Oficial de la República de Colombia. Bogotá D. C., 30 de diciembre de 2003.

Ley $\mathrm{N}^{\circ}$ 594. Por medio de la cual se dicta la Ley General de Archivos y se dictan otras disposiciones. Diario Oficial de la República de Colombia. Bogotá D. C., 14 de julio de 2000.

Resolución $\mathrm{N}^{\circ}$ 8934. Por la cual se establecen las directrices en materia de gestión documental y organización de archivos que deben cumplir los vigilados por la Superintendencia de Industria y Comercio. Diario Oficial de la República de Colombia. Bogotá D. C., 19 de febrero de 2014.

Sierra Escobar, L. F. (2012). Gestión documental enfocada a procesos: una mirada desde la administración pública distrital. Revista Interamericana de Bibliotecología, 35(3).

Torres Fragoso, J. (2008). Marco conceptual de la gerencia pública para América Latina. Cuadernos de Administración, 39(1), 19-28.

X Conferencia Iberoamericana de Ministros de Administración Pública y Reforma del Estado. (2008). Carta Iberoamericana de Calidad en la Gestión Pública, San Salvador, El Salvador. Recuperado de: http://biblioteca.icap. ac.cr/rcap/56_57/carta_ibero.pdf 